\title{
On the temporal variability of the 'strahl' and its relationship with the solar wind characteristics: Stereo SWEA observations.
}

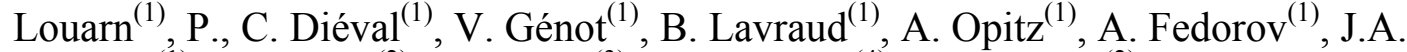

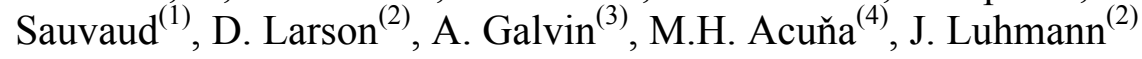

(1) Centre d'Etude Spatiale des Rayonnements, CESR, UPS/CNRS -France

(2) Space Science Laboratory, University of California, Berkeley, CA, USA

(3) Department of Physics, University of New Hampshire, NH, USA

(4) NASA Goddard Space Flight Center, Greenbelt, MD, USA

\begin{abstract}
The 'strahl' is a specific population of the solar wind, constituted by strongly field-aligned electrons flowing away from the Sun, with energies $>60 \mathrm{eV}$. Using the Solar Wind Electron Analyzer (SWEA) onboard STEREO, we investigate the short-scale fluctuations of this population. It is shown that its phase space density (PSD) at times presents fluctuations larger than $50 \%$ at scales of order of minutes and less. The fluctuations are particularly strong for periods of a few tens of hours in high speed streams, following the crossing of the Corotating Interaction Region, when the strahl is also the most collimated in pitch angle. The amplitude of the fluctuations tends to decrease in conjunction with a broadening in pitch angle. Generally, the strongly fluctuating strahl is observed when the magnetic field is also highly perturbed. That SWEA is able to perform a very rapid $3 \mathrm{D}$ analysis at a given energy is essential since it can be demonstrated that the observed magnetic turbulence can only marginally perturb the PSD measurements.
\end{abstract}

\section{Introduction}

It is well established that the electron distribution functions in the solar wind (SW) are far to be maxwellian (for early works on the subject, see Montgomery et al, (1968), Ogilvie et al (1971), Feldman et al (1975)). Four distinct electron populations are generally identified: (1) the 'core' corresponding to the bulk of the electrons, with energies below $\sim 60 \mathrm{eV}$, (2) an isotropic suprathermal population from $\sim 60 \mathrm{eV}$ to more than $1 \mathrm{keV}$ : the 'halo', (3) a strongly field-aligned suprathermal one: the 'strahl', and (4) a 'super halo' at keV energies. These populations are characterized by different slopes in their energy spectra or anisotropies with respect to the ambient magnetic field (see for example, Rosenbauer et al (1977), Feldman et al (1978), Lin (1985), Pilipp et al, 
(1987), Gosling et al (2001)). It is generally considered that the 'strahl' is formed by electrons arriving directly from the solar exobase. It constitutes the primary carrier of the SW heat flux.

The different electron populations result from a complex interplay between various physical processes involving (1) the initial coronal conditions at the exobase, (2) the SW global magnetic topology and electric potential structure, and (3) small scale processes as Coulomb scattering (mostly for the core population) and wave/particles interactions. The basic ingredient of most exospheric models is the free propagation of the particles, with conservation of the dynamic invariants. The focussing effect of the decreasing magnetic field magnitude with radial distance and the acceleration/filtering due to the large-scale electric potential tend to concentrate the electron populations in particular regions of the phase space. They are counter-balanced by microscopic processes - the Coulomb collisions in the lower corona and various wave-particle interactions all along the propagation path of the particles - that diffuse electrons in phase space and contribute to a thermal relaxation of the distribution. A considerable literature has been devoted to this subject (Jockers (1970), Gary and Feldman (1977), Scudder and Olbert (1979), Lemons and Feldman (1983), Schwartz (1980), Pilipp et al (1987), Marsch (1991), Maksimovic et al, (1997), Pierrard et al, (1999), Chen at al (2003)). The general problem is very challenging and a self-consistent theory of the dynamics and thermal relaxation of expanding collisionless magnetized plasmas does not exist yet. In particular, the fact that the strahl is much broader in pitch angle than what would be expected from the conservation of adiabatic invariants is still a matter of investigations (Maksimovic et al, (2005), Gary and Saito (2007)).

In the present paper, we discuss another aspect of the strahl, generally ignored in the literature: its short-scale variability at scales of the order of minutes and less. We propose a first investigation of the spectral and statistical properties of this 'noise' and we analyze its possible relationship with particular states of the solar wind. Our general motivation is to show that these short-scale fluctuations are an intrinsic characteristic of specific SW streams. They may provide information on their condition of formation and their energy coupling with magnetic turbulence.

\section{Instrumentation.}

The STEREO mission consists of two spacecraft that slowly drift ahead and behind the Earth on similar Solar orbits (Kaiser et al., 2008). The mission was designed to stereoscopically study solar phenomena and to study solar wind structures in a multi-spacecraft fashion. Here we primarily use data from the two solar wind electron analyzers (SWEA) (Sauvaud et al, 2008) from the IMPACT instrument suite (Luhmann et al., 2008), 
complemented by ion data from the PLASTIC instruments (Galvin et al., 2008) and magnetometers (MAG) (Acuña et al, 2008).

Identical instruments are present onboard each of the two STEREO spacecraft. The spacecraft are nonspinning due to the solar imaging requirements of the mission. The SWEA electron instruments are top-hat electrostatic analyzers with a deflection system that allows to measure incoming particles up to angles of $\pm 65^{\circ}$ from the regular top-hat entrance (that looks in the plane perpendicular to the Sun-spacecraft direction). The SWEA instruments are thus able to measure most of the electron distribution function $(\sim 80 \%$ of the $4 \pi$ solid angles) apart from two cones in the Sunward and anti-Sunward directions.

In normal operation, quasi 3D distributions are measured in $2 \mathrm{~s}$, from $1 \mathrm{eV}$ to $3 \mathrm{keV}$ in 48 energy steps (bin to 16 energy intervals before transmission). The angular resolution is $\sim 22^{\circ} \times 22^{\circ}$, which corresponds to 6 deflection angles and 16 angular sectors in azimuth. Due to telemetry restriction, 1 over 15 quasi-3D distributions is transmitted to Earth, offering a 30s time resolution. However, it is important to notice that the measurement of a 3D distribution in a given energy interval is much more rapid: about 1/8s. As discussed later, this possibility of performing fast 3D samplings will be essential for the interpretation of the observed fluctuations of the phase space density.

We will use magnetic data acquired at a cadence of $1 / 8 \mathrm{~s}$ and ion measurements at 1 minute resolution.

\section{Observation of the 'strahl' with SWEA.}

Figure 1 shows plots of various plasma parameters derived from the electron and the ion sensors during March 2007. The data from the two spacecraft are organized in two series of four panels. From the top, figure 1 first shows the SW bulk velocity (panels a and d) and the electron pitch angle distribution (panels $\mathrm{b}$ and e). We consider here electrons with energies at $\sim 250 \mathrm{eV}$, meaning in practice electrons measured from 194 to $314 \mathrm{eV}$ since the particle counts are integrated over three successive energy steps before transmission.

The strahl is well apparent in the pitch angle distribution. It corresponds to the large increase in the PSD at $0^{\circ}$ or $180^{\circ}$ that contributes to the strong pith-angle anisotropy observed more than $80 \%$ of the time. In panels c and g, we show the average PSD of this population. It is obtained by averaging the particle count rates over the $0-40^{\circ}$ or $140-180^{\circ}$ pitch angle sectors, depending on the direction of the magnetic field. The black dots correspond to the actual measurements, made at a cadence of $30 \mathrm{~s}$. The red line is a sliding window averaging of the PSD. Due to the FOV limitation, some cautions are needed to avoid inadequate determinations of the mean PSD. Indeed, even if the averaging is made over angular sectors larger than the exclusion cones, a significant 
part of the strahl might be missed when the magnetic field vector is too close to the solar direction. In the rest of the study, we choose to eliminate measurements made when the angle between the magnetic field and the solar direction is less than $20^{\circ}$. For angles smaller than $20^{\circ}$, more than half of the angular sector on which the mean PSD is calculated would be excluded.

To establish the general SW conditions, let us first consider the SW bulk velocity. Three crossings of high speed streams are evident in the figure (panels a, e). The most pronounced one starts on March 11, with an increase in velocity from 350 to $700 \mathrm{~km} / \mathrm{s}$. The crossing of the interface between the slow and the high speed stream is seen around March 12. The velocity remains large (>600 km/s) until March 17, then decreases continuously to a minimum reached on March $22(\sim 300 \mathrm{~km} / \mathrm{s})$. Two other significant increases in the SW velocity start on March 5 and 24. Their maximum velocity remains smaller than $600 \mathrm{~km} / \mathrm{s}$ and the streams themselves are crossed in $\sim 1$ day.

Many studies based on Helios, IMP and Wind data have demonstrated that encounters with high speed streams lead to important modifications in the observational characteristics of the strahl. For example, it has been established that the strahl becomes more intense and collimated during the crossing of magnetic sectors and high speed streams (Feldman et al (1978), Fitzenreiter et al, (1998), Ogilvie et al (1999)). Figure 1 gives a clear illustration of this fact. The most intense field-aligned fluxes are seen during the phases of increasing SW velocity (on March 6, 12 and 25). Later, during periods of 10-30 hours following the entering into the high speed stream, a well defined and collimated strahl is observed. The flux of the strahl then remains large for periods of several days: March 6 to 9 , March 13 to 20, March 25 to 28. It always vanishes when the velocity becomes smaller than $400 \mathrm{~km} / \mathrm{s}$ (on March 10, 20,30). This slow evolution of the flux also corresponds to a progressive broadening in pitch angles. If we consider the second high speed stream, the pitch angle width of the strahl is typically $\sim 30^{\circ}$ on March, 13 and $\sim 60^{\circ}$ on March, 20 . This broadening in pitch angle could be related to the existence of more effective diffusion mechanisms (wave/particle scattering, coulomb collision) in this trailing part of the stream, relatively far from the initial slow/fast wind transition.

We now focus the analysis on another characteristic of the strahl, well apparent in panels $\mathrm{c}$ and $\mathrm{g}$ : its intriguing large level of short scale fluctuations. As seen on March 13-14, for example, the PSD of the strahl (indicated by the black dots) is indeed occasionally very fluctuating, with a 'noise' that reaches almost $100 \%$ of the mean PSD (given by an averaging on a sliding window, in red in the plot). Interestingly, this 'noise' is not constant. The strahl seems particularly 'noisy' on March 6, 13 and 26, just after the crossings of stream interfaces. The level of the fluctuations then tends to decrease as STEREO exits the high speed streams (March 
$8,19,27)$. This evolution is best observed during the second stream. From March 14 to 19 , one can consider that the average PSD of the strahl is roughly constant. The progressive thinning of the scattered dot plot indicates that the fluctuations have decreased by typically a factor 3 during the period.

Note that the geometrical factor of SWEA is sufficiently large to guarantee that the strahl generally corresponds to count rates in individual sectors well above 100 (and even 1000 for a dense strahl). It is thus excluded that the observed fluctuations are linked to statistical variations of a few counts only.

This noise is quantitatively analyzed in panels $\mathrm{d}$ and $\mathrm{h}$ where the dynamic spectrum of the PSD is shown. These spectra are calculated by performing a Fourier transform of the PSD on a sliding window of 2 hours. Before the Fourier transform, the PSD is normalized to its average value. We thus consider $f / f_{m}$ where $f$ is the PSD and $\mathrm{f}_{\mathrm{m}}$ the averaged PSD calculated on the sliding window. The frequencies range from $0.27 \mathrm{mHz}$ to 16 $\mathrm{mHz}$. Without the normalization procedure, the largest noise would be simply seen where the PSD is the largest. The normalized spectra thus better reflect the intrinsic level of fluctuations of the PSD. They allow to accurately identifying situations of strong variability. For example, while the largest absolute fluctuations are observed when the strahl is particularly intense (on March 5 or 12, for example), it is only a few hours later that the normalized fluctuations are at maximum. For the three streams, this maximum noise is observed during the few tens hours that follow the stream interfaces (March 6-7, 13-14 and 26). The total spectral density obtained from the Fourier analysis (red line superposed to the dynamic spectra) is about a factor 3 larger in the first part of the stream than in its trailing edge (on March 8-9, 15-19 and 27-30).

Let us finally note that STEREO A and B globally observed the same periods of strongly or weakly fluctuating strahl with a delay of a few hours. This is expected since the two spacecraft are relatively close in March 2007, with a typical separation of $2^{\circ}$ in solar longitude. A closer inspection reveals that the level of the fluctuations may locally present significant differences. For example, on March 17, STEREO-B sees a burst of fluctuations, not observed by STEREO-A. The opposite occurs on March 26, a noise increase is measured by STEREO-A but not by STEREO-B. A more precise comparison would require a fine inter-calibration of the instruments. Nevertheless, these variations from one spacecraft to the other may reveal some fine structuring and/or local energy exchanges in the flow. This interesting possibility will deserve further analysis.

\section{Relation with magnetic fluctuations.}

In figure 2, complementary aspects of the fluctuations are presented. The analysis is focussed on the period March 11-20. The three upper panels show, as in figure 1, (a) the SW bulk velocity, (b) the electron pitch-angle 
distribution $(250 \mathrm{eV})$, and (c) the PSD of the strahl (here calculated on the $0-22^{\circ}$ pitch angle sector bin). In panel $d$, the normalized fluctuations of the PSD are displayed. This quantity is given by $\left.\left(f-f_{m}\right) / f_{m}\right)$ where $f_{m}$ is, as previously, the averaged PSD calculated on 2 hours intervals. The normalized fluctuations are then used to compute the density functions presented in panel e. To obtain this quantity, we consider the normalized fluctuations observed during a 4 hours sliding window and bin them as a function of their amplitude with 40 intervals in amplitude, covering fluctuations from 0 to 1 (relative to the mean PSD). The resulting density functions are presented vertically in colour code. This plot shows the proportion of fluctuations with given amplitude observed during a 4 hours period centred on the considered time. Red pixels correspond to a proportion of $\sim 10 \%$, yellow $\sim 5 \%$ and green $\sim 2 \%$. This plot basically presents in a more quantitative and objective way the visual appearance of strahl variability seen in panel d. It should be noted that modifying the duration of the sliding window, for example from 1 hours to 8 hours, does not significantly modify the main features observed in the plot.

The significant increase in the proportion of large amplitude fluctuations is obvious from March 13 to March $14 \sim 1200$ UT. The black line indicates the limit in amplitude below which $2 / 3$ of the fluctuations are observed. During the March 13-14 period, we see that more than 1/3 of fluctuations are larger than 1/4 of the average PSD $\left(\left(\mathrm{f}-\mathrm{f}_{\mathrm{m}}\right)>0.25 \mathrm{f}_{\mathrm{m}}\right)$. After March $141200 \mathrm{UT}$, the percentage of large amplitude fluctuations decreases continuously. Finally, on March 19 and 20, a negligible proportion of fluctuations reach 0.25 . This figure shows precisely how the variability of the strahl evolves in the high speed stream. Although the speed remains large from March 14 to 15, the most fluctuating strahl is observed during the first half of the stream.

In the two last panels of figure 2, time series of the radial component of the magnetic field (the component along the solar direction) and its dynamic spectra, from $\sim 1$ to $50 \mathrm{mHz}$, are displayed. The total spectral density of the magnetic fluctuations is also presented (red curve in panel h). It is clear that the variability of the strahl follows the general evolution of the magnetic spectral density. The magnetic field is particularly turbulent from March 12 to 14 when the strahl strongly fluctuates. The level of magnetic fluctuations also tends to decrease until March 20, like the level of the strahl fluctuations. We get the same conclusion from the analysis of other time periods and conclude that a turbulent magnetic field is likely a necessary condition for the observation of a strongly fluctuating strahl. However, it should be noted that the correlation is not always perfect. For example, just at the beginning of March 14, the level of magnetic fluctuations decreases by almost a factor $\sim 2$ when precisely the strahl fluctuations are maximum. This indicates that more subtle secondary structures may exist in the magnetic/strahl fluctuation relationships. 
Before reaching a firm conclusion, the possible perturbing effect of magnetic field fluctuations on SWEA measurements has to be estimated. Large and rapid magnetic fluctuations may affect the determination of the pitch angle distribution and the fluctuations might then be a measurement artefact. In the case of SWEA and considering a given energy step, the strahl could be inaccurately measured at this given energy if the magnetic field direction 'jumps' from one sector of deflection to the other during a sampling of the distribution. For a given energy step, a $360^{\circ} \times 120^{\circ}$ measurement is made in about $1 / 8 \mathrm{~s}$, corresponding to the analysis of 6 deflection sectors of $\sim 20^{\circ}$, with a sampling time of $\sim 20 \mathrm{~ms}$ per sector. Accurate measurements of the pitch angle distribution are guaranteed if the magnetic field orientation remains in the same deflection sector during the entire energy step. This will be the case if the magnetic field orientation varies significantly less than $20^{\circ}$ in $1 / 8 \mathrm{~s}$. Variations of $20^{\circ}$ in $1 / 8 \mathrm{~s}$ correspond to fluctuations of about $30 \%$ of one component of the field in a time scale considerably shorter than the ion gyroperiod (typically: 10-20s). This would correspond to a huge level of high frequency turbulence, actually not observed in March 2007.

We check this point by computing the variations of the magnetic field orientation observed at the normal time resolution available with STEREO, which precisely corresponds to $1 / 8$ s (figure 3). Ideally, to be sure that the magnetic field orientation does not jump from one sector to the other during the sampling, the time resolution of the measurements need to be better than $1 / 8 \mathrm{~s}$. Nevertheless, as shown below, we record a very small proportion of variations in the magnetic orientation that exceed $2-3^{\circ}$ over $1 / 8 \mathrm{~s}$ so that we can safely conclude that the magnetic fluctuations cannot significantly perturb the pitch angle measurements. To get this conclusion, we consider the magnetic field measured in March 13 (690,000 samples) and compute the fluctuations of its orientation. As seen in the figure (panel a), they hardly exceed $5^{\circ}$ even during this time period corresponding to a particularly turbulent field. More quantitatively, the histogram of the fluctuations in direction are presented below (panel b), showing that the proportion of fluctuations larger than $1^{\circ}$ is small (this is hardly visible in the upper plot given the number of points). It is also possible to calculate the probability of jump from one deflector to the other during the sampling. If $\Delta \alpha$ is the fluctuation in angle, a jump in deflection sector will occur if the initial magnetic angle is at less than $\Delta \alpha$ from the deflector limits. Assuming that the orientation of the magnetic field is randomly distributed, the probability of such an event is $\sim \Delta \alpha / 20^{\circ}\left(20^{\circ}\right.$ is the aperture of the deflection sector). Multiplying the histogram by this quantity, one thus gets the probability of jumps. It is displayed in panel c. In total the probability of jumps is less than $3 \%$. This is more than one order of magnitude less than the observed proportion of strong fluctuations of the PSD. We thus conclude that the magnetic field fluctuations observed on March 13 can only marginally affect the SWEA pitch angle measurements of the strahl. 
It is worth to mention that we take here advantage of the very rapid quasi-3D analysis made by SWEA. At a given energy, the quasi 3D determination of the distribution is made in $1 / 8 \mathrm{~s}$, thus, at least a factor $40-50$ more rapidly than what can be done with instruments that use the spin of the spacecraft to span $4 \pi$. With $3 \mathrm{D}$ measurements done in a few seconds, it would be almost impossible to demonstrate that the variability is not a measurement artefact linked to the magnetic fluctuations.

\section{Discussion and Conclusion}

The present work has to be considered as a preliminary analysis of a rarely discussed aspect of the strahl: its strong and variable level of fluctuations. Using SWEA observations, we present the phenomena and analyze its relationship with other characteristics of the solar wind. In particular, we show that the strahl is particularly fluctuating in the region of the high speed streams that immediately follows the main corotating interaction region, when it is also the most collimated in pitch angle. In the trailing part of the high speed stream, when the SW velocity decreases, the strahl is less fluctuant and becomes broader in pitch angle. We also demonstrate the close relationship between a strongly fluctuating strahl and a turbulent magnetic field.

These observations suggest that the variability of the strahl could be a characteristic parameter of the flow (as the density and the pitch angle extension of the strahl). To some extents, it could serve to define specific substructures in the stream, not necessarily apparent from other parameters. The fluctuations of the strahl could also contain information concerning the turbulent state at the exobase, above the coronal hole. However, it would be surprising if the strong magnetic turbulence, most certainly alfvénic, does not interact with the electron population. If part of this turbulence corresponds to kinetic Alfven waves, a fluctuating parallel electric field may exist along the magnetic field (as observed in the auroral zone Louarn et al, (1994), Génot et al, (2000)), and energetically interacts with the electrons. Whether the strahl fluctuations are the result of local processes, possibly distributed all along the path of the electrons, or a remnant of a turbulent state of the exobase will be the subject of future analysis. 


\section{References}

Acuňa, M.H., D. Curtis, J.L. Scheifele, C.T. Russell, P. Schroeder, A. Szabo, J.G. Luhmann, The STEREO/IMPACT magnetic field experiement, Space Science Reviews, Volume 136, DOI: 10.1007/s11214007-9259, 2007.

Chen, Y., R. Esser, and Y.Q. Hu, Numerical modeling of the halo electrons in the fast solar wind, J. Geophys. Res., 108, 1371, doi:10.1029/2003JA009957, 2003.

Feldman, W. C., et al., Solar wind electrons, J. Geophys. Res., 80, 4181-4196, 1975.

Feldman, W. C., et al., Characteristic electron variations across simple high-speed solar wind streams, $J$. Geophys. Res., 83, 5285-5295, 1978.

Fitzenreiter, R. J., K. W. Ogilvie, D. J. Chornay, and J. Keller, Observations of electron velocity distribution functions in the solar wind by the WIND spacecraft: High angular resolution strahl measurements, Geophys. Res. Lett., 25, 249-252. 1998.

Galvin et al, The Plasma and Suprathermal Ion Composition (PLASTIC) Investigation on the STEREO observatories, Space Science Reviews, Volume 136, DOI: 10.1007/s11214-007-9296-x, 2007.

Gary, S. P., and W. C. Feldman, Solar wind heat flux regulation by the whistler instability, J. Geophys. Res., 82, 1087-1094, 1977.

Gary, P.S. and S. Saito, Broadening of solar wind strahl pitch angles by the electron/electron instability: Particle in cell simulations. Geophys. Res. Lett., 34, L1411, doi:10.1029/2007GL030039, 2007.

Génot V., P. Louarn and F. Mottez, Electron acceleration by Alfven waves in density cavities, J. Geophys. Res., 105, 27611, 2000.

Gosling, J. T., R. M. Skoug, and W. C. Feldman, Solar wind electron halo depletions at $90^{\circ}$ pitch angle, Geophys. Res. Lett., 28, 4155-4158, 2001.

Jockers, K., Solar wind models based on exospheric theory, Astron. Astrophys., 6, 219-239, 1970.

Kaiser, M.L., T.A. Kucera, J. M. Davila, O.C. St Cyr, M. Guhathakurta, E. Cristian, The STEREO mission: an introduction, Space Science Reviews, Volume 136, DOI: 10.1007/s11214-007-9277-0, 2007.

Lemons, D.S. and W. C. Feldman, Collisional modification to the exospheric theory of solar wind halo electron pitch angle distribution, J. Geophys. Res., 88, 6881, 1983.

Lin, R. P, Energetic solar electrons in the interplanetary medium, Solar Phys., 100, 537-561. 1985.

Louarn P., J.E. Wahlund, T. Chust, H. de Féraudy, A. Roux, B. Holback, P. Dovner, A. Eriksson and G. Holmgren, Observation of kinetic Alfven waves by the Freja Spacecrat, Geophys. Res. Lett., 21, 1847, 1994.

Luhmann, J. D., at al, STEREO IMPACT investigation goals, measurements and data products overview, Space Science Reviews, Volume 136, DOI: 10.1007/s11214-007-9170-x, 2007.

Maksimovic, M., V. Pierrard and J.Lemaire, A kinetic model of the solar wind with Kappa distribution functions in the corona, Astronomy \& Astrophysics, 324, 725-734, 1997

Maksimovic, M. , et al. , Radial evolution of the electron distribution functions in the fast wind between 0.3 and 1.5 A.U., J. Geophys. Res., 110, A09104, doi:10.1029/2005JA011119, 2005.

Marsch, E., Kinetic physics of the solar wind plasma, in Physics of the inner heliosphere II, Particles, Waves and Turbulence, edited by R. Schwenn and E. Marsch, p. 45, Springer-Verlag, New York, 1991.

Montgomery, M. D., S. J. Bame, and A.J.Hundhausen, Solar wind electrons, Vela 4 measurements, J. Geophys. Res., 73, 4999, 1968. 
Ogilvie, K. W., J. D. Scudder, and M. Sugiura, Electron flux in the solar wind, J. Geophys. Res., 76, 8165, 1971.

Ogilvie, K. W., L. F. Burlaga, D;J. Chornay, and R.J. Fitzenreiter, Sources of the solar wind electron strahl in 1995, J. Geophys. Res., 104, 22389, 1999.

Pierrard, V., M. Maksimovic, and J. Lemaire, Electron velocity distribution functions from the solar wind to the corona, J. Geophys. Res., 104, 17,021-17,032, 1999.

Pilipp, W. G., et al., Characteristics of electron velocity distribution functions in the solar wind derived from the Helios plasma experiment, J. Geophys. Res., 92, 1075-1092, 1987.

Rosenbauer, H., et al., A survey on initial results of the Helios plasma experiment, J. Geophys. Res., 42, 561$580,1977$.

Sauvaud et al, The IMPACT Solar Wind Electron Analyzer (SWEA), (2007), Space Science Reviews, Volume 136, DOI: $10.1007 / \mathrm{s} 11214-007-9146-6$

Schwartz, S. J., Plasma instabilities in the solar wind: A theoretical review, Rev. Geophys., 18, 313-336, 1980.

Scudder, J. D., and S. Olbert, A theory of local and global processes which affect solar wind electrons, 1, The origin of typical 1 AU velocity distribution functions-Steady state theory, J. Geophys. Res., 84, 2755-2772, 1979. 


\section{Figure captions}

Figure 1. Plasma parameters measured by SWEA and the ion sensor during March 2007. For each spacecraft, 4 panels are shown, form top: (a) SW bulk velocity, (b) pitch angle distribution of the $250 \mathrm{eV}$ electrons,(c) mean PSD of the stral and (d) dynamic spectra of the fluctuations of the stral PSD

Figure 2. Comparison between SWEA and magnetic measurements (March 12-20). From top: (a) SW bulk velocity, (b) pitch angle distribution of the $250 \mathrm{eV}$ electrons, (c) mean PSD of the stral. In the two panels below: (d) normalized fluctuation of the strahl and (e) density function of the fluctuations. Red is for the largest proportion of fluctuation (10 $\%$, green and blue the smallest (below $2 \%$ ). In the two lowermost panels: (f) radial component of B and (g) associated dynamic spectra.

Figure 3. (a) time plot of the fluctuations of the magnetic orientation, (b) Hodogram of the variations of the orientation of the magnetic field and (c) probability of jumps of deflection angle during a sampling. 
Solar Wind Bulk Velocity - Ahead

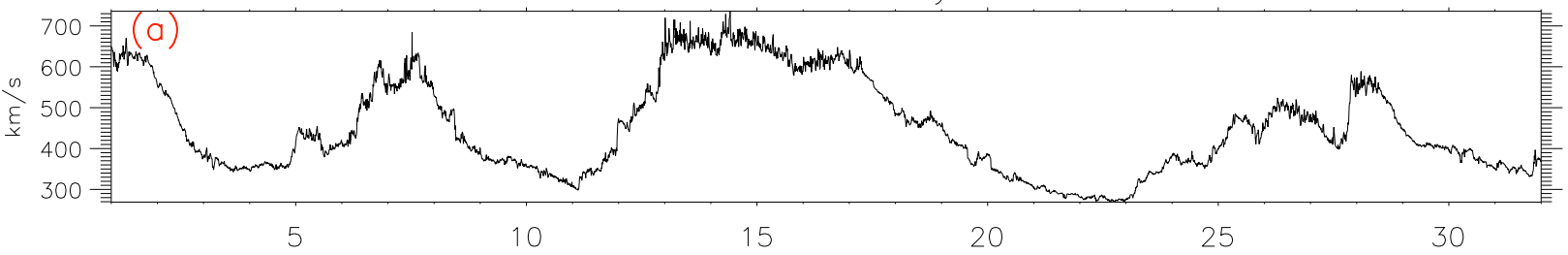

Pitch angle distribution (250 eV)

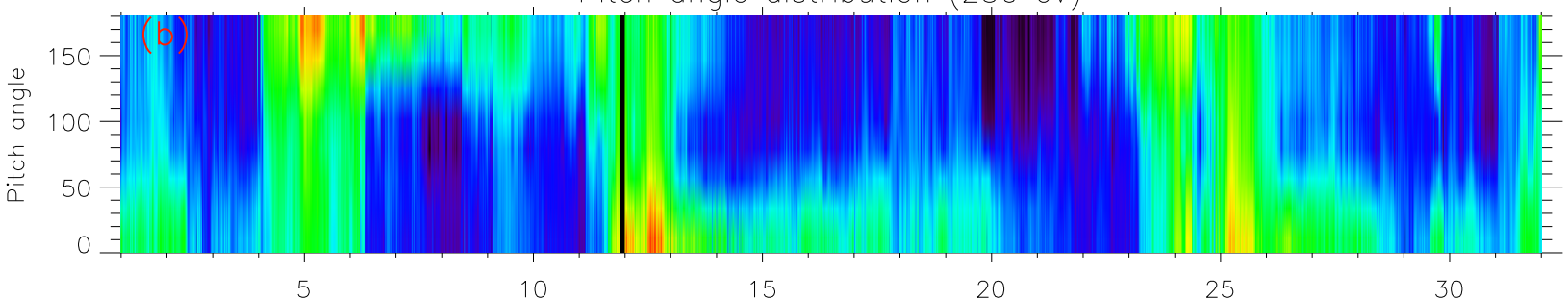

Averaged Distribution (para/antipara - 45 deg.)
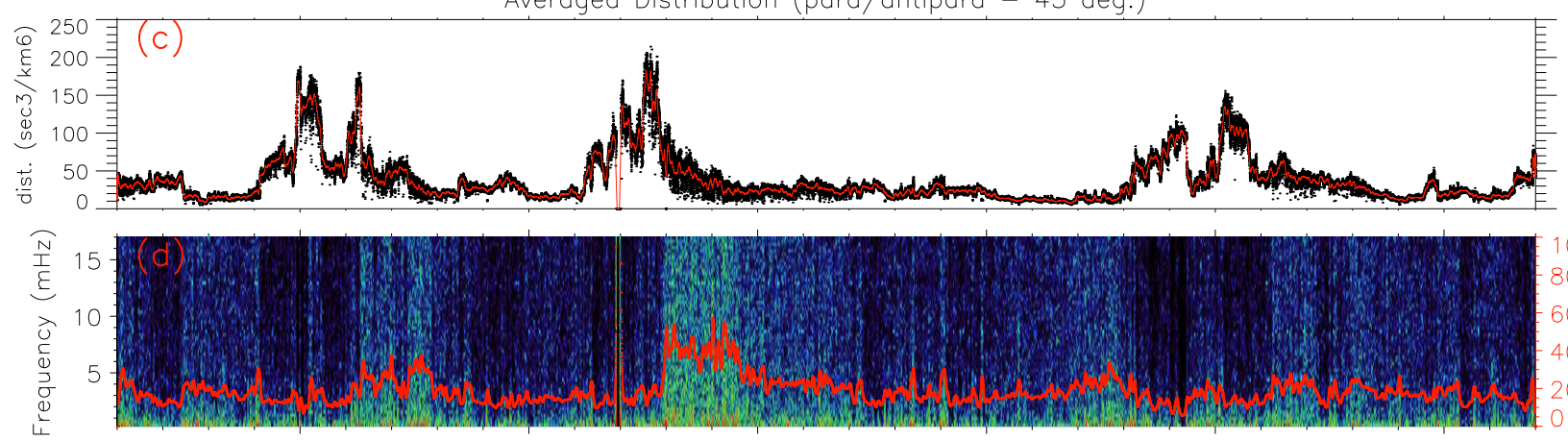

$\begin{array}{lllll}10 & 15 & 20 & 25 & 30\end{array}$

Solar Wind Bulk Velocity - Behind

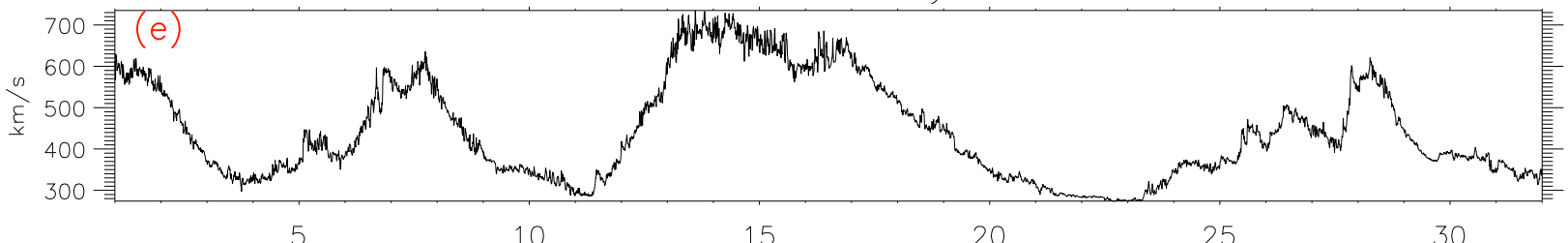

Pitch angle distribution (250 eV)

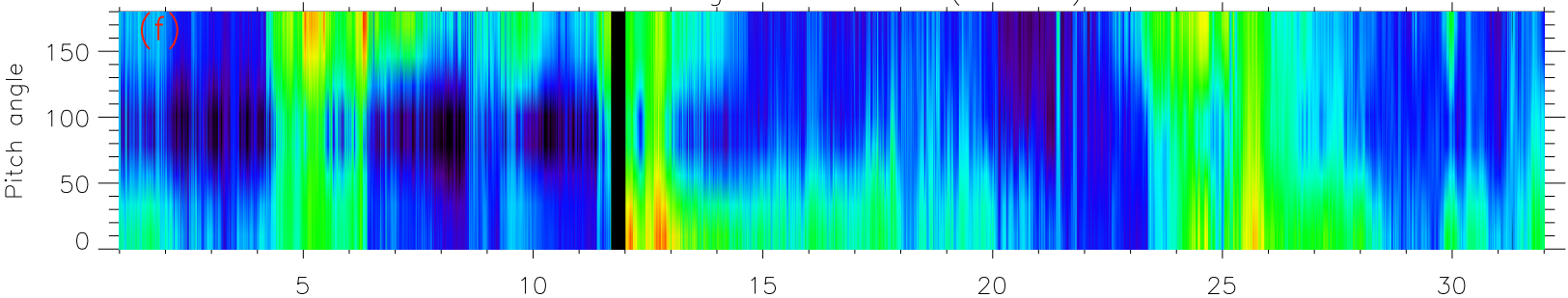

Averaged Distribution (para/antipara - 45 deg.)
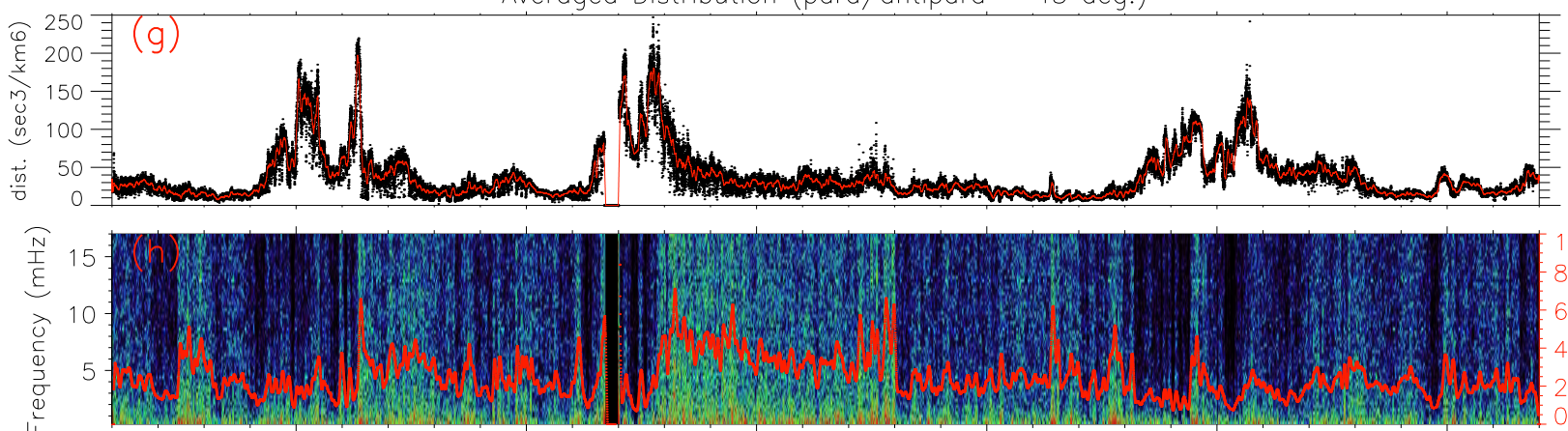

$5-10$

10

15

20

25

30

Day in MARCH 2007 
AHEAD
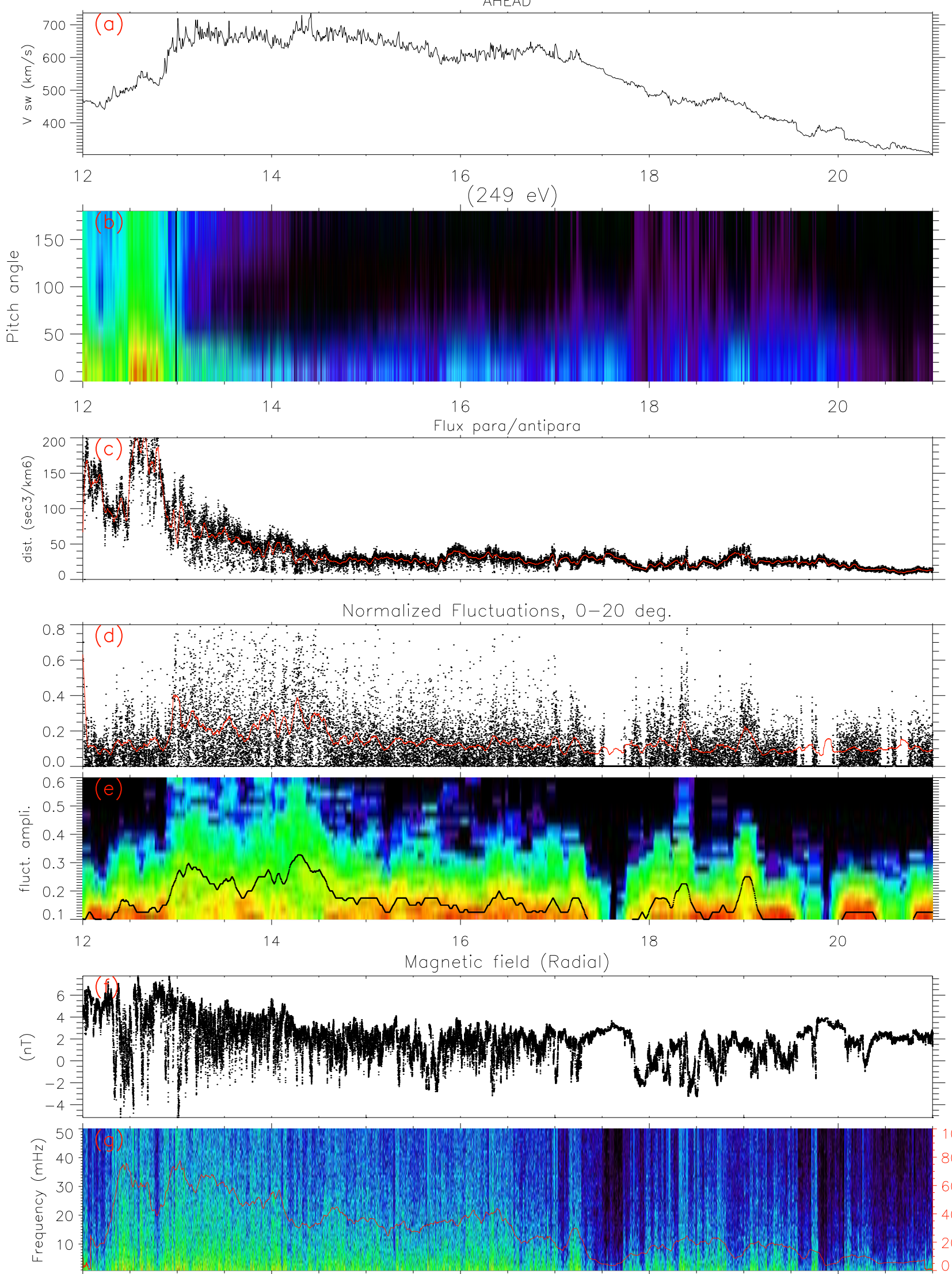

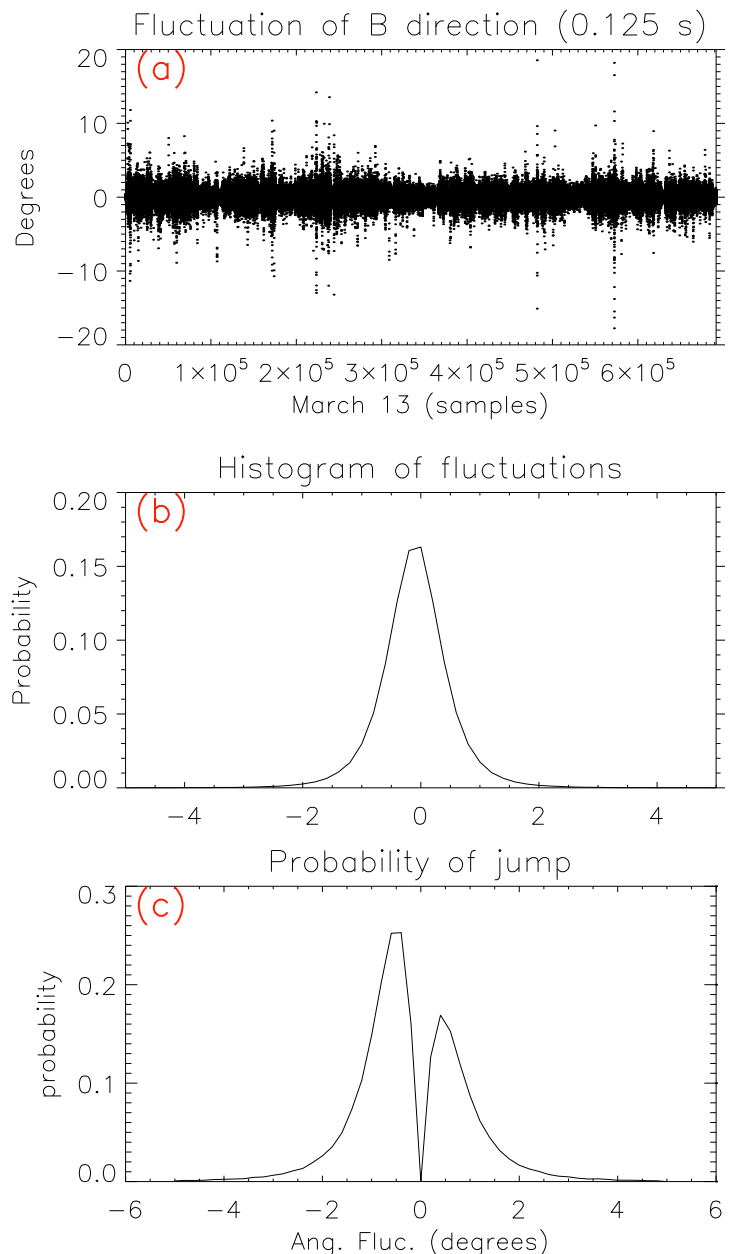